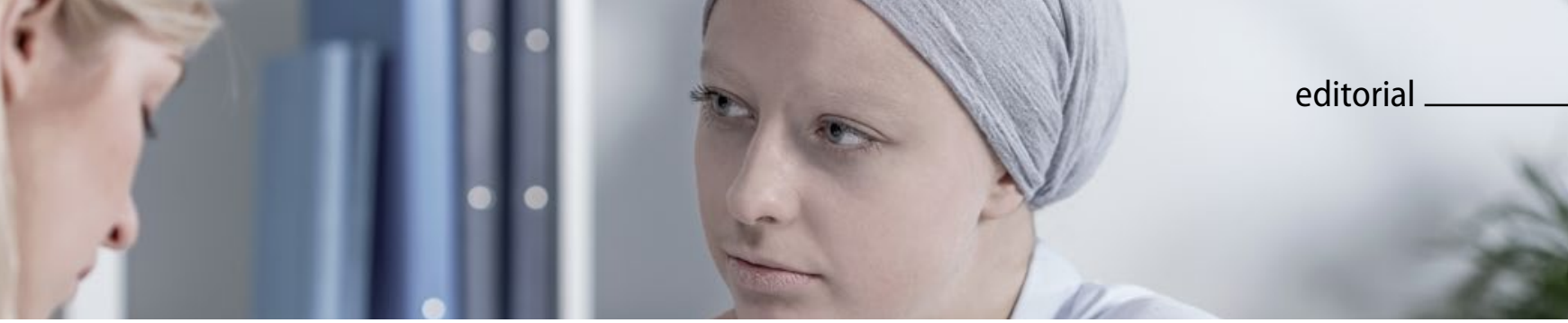

Patientencoaching in der Onkologie

\title{
Mehr Zeit für die Patientenbetreuung durch Delegation
}

Tumorerkrankungen sind nach den Herz-/Kreislauferkrankung die zweithäufigste Todesursache in Deutschland. Die Zukunft der Versorgung der Tumorpatienten steht vor einer großen Herausforderung: Der zunehmenden Prävalenz von Krebserkrankungen. Letzteres hängt wesentlich von zwei Aspekten ab:

1. Von der demografischen Entwicklung. Die Inzidenz der Tumorerkrankung nimmt mit dem Alter zu und der Anteil der älteren Menschen in der Bevölkerung nimmt zu.

2. Von den medizinischen Fortschritten in der Behandlung der Tumorerkrankungen.

Beide Faktoren führen bei vielen Patienten zu einer Chronifizierung der Tumorerkrankung. Im gleichen Maß, wie der Betreuungsaufwand für die komplexen Therapien in einem vom sozialen Umfeld und Komorbiditäten des Patienten geprägten Behandlungsablauf zunimmt, nimmt die ärztliche Zeitressource für die fachärztliche Betreuung der Patienten ab. Schon jetzt ist absehbar, dass im Bereich der fachärztlichen hämatoonkologischen Versorgung eine Mangelsituation entsteht. Neue Betreuungsmodelle sind deshalb unabdingbar.

\section{Patient als Experte seiner eigenen Therapie}

Eine gute Betreuung des Patienten berücksichtigt die patientenindividuellen Ressourcen auf dem Weg durch die Tumortherapie. So lassen sich Defizite aufspüren und ggf. gezielt intervenieren, was den Erfolg der Therapie verbessert. Eine gute Behandlung bedeutet dabei nicht nur das Management der therapieassoziierten Toxizitäten und Nebenwirkungen, sondern sollte auch ein holistisches Konzept bei der Patientenversorgung sein. Dabei müssen sowohl soziale als auch psychische und kognitive Aspekte der älter werdenden Generation mit erfasst werden. Die Onkologie wird zunehmend ambulanter und viele Onkologika können mittlerweile oral verabreicht werden. Dies bedeutet aber, dass wir den Patienten - soweit möglich - zu einem Experten seiner Therapie machen müssen, um die Qualität der Versorgung zu gewährleisten. Betreuungsansätze müssen noch mehr als in der Vergangenheit in das private Umfeld des Patienten integriert werden. Dies setzt einen genauen und individuellen Einblick in die Lebenssituation des Patienten voraus. Verhalten lässt sich nur in Beziehungen nachhaltig beeinflussen. Die Teamressource kann schon heute dabei genutzt werden.

\section{Patientencoaching als neues Betreuungsmodell}

Der Patientencoach ist ein innovatives Betreuungsmodell, welches auf der Delegation nichtärztlicher Leistung an eine geschulte Pflegekraft (Patientencoach) beruht. Der Patientencoach

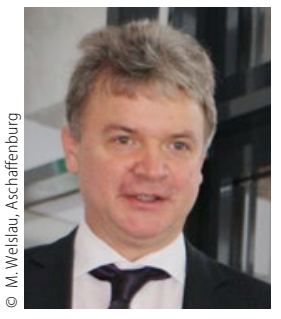

Dr. Manfred Welslau

Onkologie Aschaffenburg

m.welslau@onkologie-ab.de

begleitet den Patienten in Ergänzung zum therapieführenden Arzt in strukturierten und bedarfsorientierten Terminen durch die Therapie. Das strukturierte Coachgespräch liefert dabei

- Hilfe zur Selbsthilfe,

- Anleitungsmöglichkeiten, selbst etwas gegen bestimmte Symptome zu tun,

- Subjektive Bedeutung der Therapie für den Alltag des Patienten,

- Besserung der Selbstwirksamkeit und

- Verbesserung der Lebensqualität des Patienten.

Bei einer durchschnittlichen Zeitinvestition von 60 Minuten pro Tumorpatient sind in einem strukturierten Gespräch diese Verbesserungen zu erreichen. In der PACOCT-Studie, die auf der Plattform des WINHO durchgeführt wurde und die im letzten Jahr auf dem Jahreskongress der American Society of Clinical Oncology (ASCO) vorgestellt wurde, konnten durch ein Patientencoaching in Ergänzung zur fachärztlichen Betreuung eine Reihe an positiven Befunden erzielt werden [Welslau $\mathrm{M}$ et al. ASCO. 2016;Abstr 6517]; dazu zählten: Das therapiebezogene Wissen des Patienten wurde verbessert, weniger Patienten brachen ihre Therapie ab, die Selbstwirksamkeitsempfindung der Patienten war tendenziell besser und auch die „patient-reported outcomes“, d.h. die Therapieergebnisse aus Sicht des Patienten, fielen besser aus.

Inzwischen entstehen Fortbildungscurricula für das neue Berufsbild des Patientencoaches, Zertifizierungen der Landesärztekammern Hessen und Nordrhein liegen vor.

Die Einführung des Patientencoachings kann helfen, den Onkologen zu entlasten und unsere vordringliche Aufgabe zu optimieren: Wir müssen uns um unsere krebskranken Patienten kümmern.

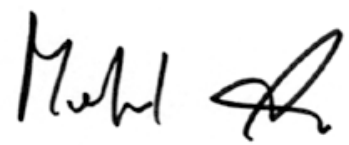

